\title{
Asymptotic Behavior of Solutions to Certain Nonlinear Schrödinger-Hartree Equations ${ }^{\star}$
}

\author{
R. T. Glassey
}

Department of Mathematics, Indiana University, Bloomington, Indiana 47401, USA

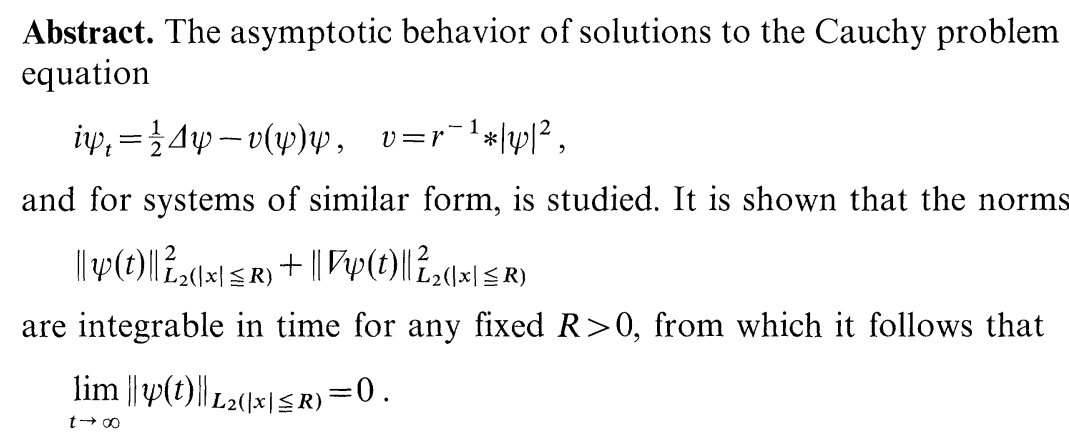

Nevertheless, it is established that an $L_{2}$-scattering theory is impossible.

\section{Introduction}

We consider classical solutions to the Cauchy problem for the equations

$$
\begin{aligned}
i \psi_{t} & =\frac{1}{2} \Delta \psi-v(\psi) \psi \quad\left(x \in \mathbb{R}^{3}, t>0\right) \\
v(\psi) & =r^{-1} *|\psi|^{2}=\int_{\mathbb{R}^{3}}|x-y|^{-1}|\psi(y, t)|^{2} d y \quad(r=|x|)
\end{aligned}
$$

and

$$
i \partial \psi_{j} / \partial t=\frac{1}{2} \Delta \psi_{j}-\sum_{k=1}^{N}\left(\psi_{j} v_{k}-\psi_{k} v_{j k}\right) \quad(j=1,2, \ldots, N)
$$

where

$$
v_{j k}=r^{-1} * \psi_{j} \psi_{k}^{-}, \quad v_{k}=v_{k k}=r^{-1} *\left|\psi_{k}\right|^{2} .
$$

Equations (1), (2) are Coulomb-free versions of the time-dependent Hartree and Hartree-Fock equations. In [2] we have treated the existence question for

* $\quad$ Research supported in part by National Science Foundation Grant GP 37630 
Equations (1), (2) with coulomb terms present, and have shown that global solutions exist with the quantity [for (2)]

$$
\sum_{j=1}^{N}\left\{\left\|\psi_{j}(t)\right\|_{2}^{2}+\left\|\nabla \psi_{j}(t)\right\|_{2}^{2}\right\}
$$

remaining uniformly bounded. The notation here is

$$
\|\psi(t)\|_{2}=\left(\int_{\mathbb{R}^{3}}|\psi(x, t)|^{2} d x\right)^{1 / 2},
$$

etc. A similar result is valid for solutions to (1).

In this paper we shall obtain the following results : Let $\psi$ be a solution of (1) with finite energy norm (as above). Then for every fixed $R>0$ we have

$$
\int_{0}^{\infty} \int_{|x| \leqq R}\left(|\psi|^{2}+|\nabla \psi|^{2}\right) d x d t<\infty
$$

from which we conclude that

$$
\lim _{t \rightarrow \infty} \int_{|x| \leqq R}|\psi|^{2} d x=0 .
$$

For spherically symmetric solutions $\psi_{j}$ of (2) with finite energy, the same results are valid for each $\psi_{j}, 1 \leqq j \leqq N$. However, we also show that an $L_{2}$-scattering theory for non-trivial solutions of (1) is impossible. It is plausible that solutions to (1) do decay uniformly to zero as $t \rightarrow \infty$, but if so, the rate of decay cannot be fast enough to insure the existence of asymptotic free states.

The desired estimates follow from an identity obtained essentially through use of the multiplier $\partial \psi^{-} / \partial r$ [for (1)]. The resulting estimate is the exact analogue of Morawetz' estimate [5]. During the preparation of this work, the author learned that this multiplier was found independently and, in fact, first, by Lin. In his thesis [4], Lin studies the asymptotic behavior of solutions to equations of the form

$$
i u_{t}=\Delta u-h(x) q\left(|u|^{2}\right) u
$$

and shows that

$$
\|u(t)\|_{\infty}=0\left(t^{-3 / 2}\right) \quad \text { as } \quad t \rightarrow \infty
$$

under certain conditions on $h$ and $q^{1}$. In addition, decay of the "local energy norm" is established, and a scattering theory is developed.

The reason for the restriction to spherically symmetric solutions of (2) involves the use of a radial derivative as a multiplier; this will be evident from the proof.

Although we dealt in [2] only with generalized solutions, it is easy to see that in the absence of coulomb terms [i.e. for (1), (2)], solutions will be smooth if the data is. By induction on $k$ we can show that the norms $\sum_{|\alpha| \leqq k}\left\|D^{\alpha} \psi\right\|_{2}$ are finite for all $t \geqq 0$. For $k \leqq 2$, this was done in [2]. For higher values of $k$, we write the potential $v$ [in the case of Equation (1)] as

$$
v=\int_{\mathbb{R}^{3}}|z|^{-1}|\psi(x-z, t)|^{2} d z
$$

1 The restrictions involving $h$ can be removed, e.g. $h \equiv 1$ is admissible (private communication from Prof. Walter Strauss) 
and take successively higher derivatives of $|\psi|^{2}$. The induction suceeds easily when we take into account the inequality

$$
\int|x-y|^{-2}|\varphi(y)|^{2} d y \leqq 4 \int_{\mathbb{R}^{3}}|\nabla \varphi|^{2} d x \quad\left(\varphi \in C_{0}^{\infty}\left(\mathbb{R}^{3}\right)\right)
$$

and apply Gronwall's inequality.

\section{Time Decay}

We shall work first with Equations (2); the corresponding results for (1) can be simply deduced from this. The system to be studied is

$$
i \partial \psi_{j} / \partial t=\frac{1}{2} \Delta \psi_{j}-\sum_{k=1}^{N}\left(\psi_{j} v_{k}-\psi_{k} v_{j k}\right) \quad(j=1,2, \ldots, N)
$$

for $x \in \mathbb{R}^{3}, t>0$. Smooth initial values $\psi_{j}(x, 0)=\varphi_{j}(x)$ are given, which belong, say, to $\mathscr{S}$.

Here

$$
v_{j k}=\int|x-y|^{-1} \psi_{j} \psi_{k}^{-} d y, \quad v_{k}=\int|x-y|^{-1}\left|\psi_{k}\right|^{2} d y
$$

so that $v_{j k}{ }^{-}=v_{k j}$. From [2] we have that there is a constant $M$ such that the energy satisfies

$$
\sum_{j=1}^{N}\left[\left\|\psi_{j}(t)\right\|_{2}^{2}+\left\|\nabla \psi_{j}(t)\right\|_{2}^{2}\right] \leqq M
$$

for all $t \geqq 0$.

Let $\zeta=\zeta(r), r=|x|$, be a smooth bounded real-valued function. We obtain our estimates from the following identity:

Lemma. Let $\psi_{j}, j=1,2, \ldots, N$, be solutions to (2) with finite energy. Then

$$
\begin{aligned}
& -\frac{d}{d t} \operatorname{Im} \sum_{j=1}^{N} \int \zeta \psi_{j} \partial \psi_{j}-\partial r d x=-\sum_{j=1}^{N} \int \zeta^{\prime}\left|\partial \psi_{j} / \partial r\right|^{2} d x \\
& -\sum_{j=1}^{N} \int \zeta|x|^{-1}\left(\left|\nabla \psi_{j}\right|^{2}-\left|\partial \psi_{j} / \partial r\right|^{2}\right) d x-2 \pi \sum_{j=1}^{N} \zeta(0)\left|\psi_{j}(0, t)\right|^{2} \\
& +\sum_{j=1}^{N} \int\left|\psi_{j}\right|^{2}\left[\frac{1}{2} \Delta \zeta|x|^{-1}-\zeta^{\prime}|x|^{-2}+\frac{1}{4} \Delta \zeta^{\prime}\right] d x \\
& +\sum_{k, j=1}^{N} \int \zeta\left[\left|\psi_{j}\right|^{2}\left(\partial v_{k} / \partial r\right)-\operatorname{Re} \psi_{j}-\psi_{k} \partial v_{j k} / \partial r d x\right.
\end{aligned}
$$

We sketch the derivation of the lemma. We begin by multiplying each of Equations (2) by $\zeta \partial \psi_{j}^{-} / \partial r$ (the use of $\zeta$ is an idea from $[8,9]$ ). The real part of the resulting expression is then summed over $j$ and integrated over $\mathbb{R}^{3}$. The result can be written as

$$
I_{1}=I_{2}+I_{3}
$$


where

$$
\begin{aligned}
& I_{1}=i \int \sum_{j=1}^{N} \sum_{l=1}^{3} \zeta x_{l}|x|^{-1}\left(\frac{\partial \psi_{j}^{-}}{\partial x_{l}} \frac{\partial \psi_{j}}{\partial t}-\frac{\partial \psi_{j}}{\partial x_{l}} \frac{\partial \psi_{j}^{-}}{\partial t}\right) d x \\
& I_{2}=\frac{1}{2} \sum_{j=1}^{N} \sum_{l=1}^{3} \int \zeta|x|^{-1} x_{l}\left(\frac{\partial \psi_{j}^{-}}{\partial x_{l}} \Delta \psi_{j}+\frac{\partial \psi_{j}}{\partial x_{l}} \Delta \psi_{j}^{-}\right) d x \\
& I_{3}=-2 \sum_{k, j=1}^{N} \sum_{l=1}^{3} \int \zeta|x|^{-1} x_{l}\left[\operatorname{Re}\left(\frac{\partial \psi_{j}^{-}}{\partial x_{l}} \psi_{j} v_{k}\right)-\operatorname{Re}\left(\frac{\partial \psi_{j}^{-}}{\partial x_{l}} \psi_{k} v_{j k}\right)\right] d x
\end{aligned}
$$

We rewrite $I_{1}$ as

$$
I_{1}=i \int \sum_{j=1}^{N} \sum_{l=1}^{3} \zeta|x|^{-1} x_{l}\left[\frac{\partial}{\partial x_{l}}\left(\psi_{j}^{-} \frac{\partial \psi_{j}}{\partial t}\right)-\frac{\partial}{\partial t}\left(\frac{\partial \psi_{j}}{\partial x_{l}} \psi_{j}^{-}\right)\right] d x
$$

and integrate by parts; in doing so we find that

$$
I_{1}=(d / d t) \operatorname{Im} \int \sum_{j=1}^{N} \zeta \psi_{j}\left(\partial \psi_{j} / \partial r\right) d x-\operatorname{Re}\left(i \int \sum_{j=1}^{N}\left(\zeta^{\prime}+2|x|^{-1} \zeta\right) \psi_{j}^{-} \partial \psi_{j} / \partial t d x\right)
$$

The second term appearing here may be calculated by appealing to the Equation (2) again. Integrating by parts several more times, we evaluate $I_{1}$ as

$$
\begin{aligned}
I_{1}= & \frac{d}{d t} \operatorname{Im} \int \sum_{j=1}^{N} \zeta \psi_{j}-\partial \psi_{j} / \partial r d x \\
& +\int \sum_{j, k=1}^{N}\left(\zeta^{\prime}+2|x|^{-1} \zeta\right)\left(\left|\psi_{j}\right|^{2} v_{k}-\operatorname{Re} \psi_{j} \overline{\psi_{k}} v_{j k}\right) d x \\
& +\frac{1}{2} \int \sum_{j=1}^{N}\left(\zeta^{\prime}+2|x|^{-1} \zeta\right)\left|\nabla \psi_{j}\right|^{2} d x \\
& -\frac{1}{4} \int \sum_{j=1}^{N}\left|\psi_{j}\right|^{2}\left(\Delta\left(\zeta^{\prime}+2|x|^{-1} \zeta\right)\right) d x .
\end{aligned}
$$

Again integrating by parts, we calculate $I_{2}$ in a straightforward fashion and obtain

$$
I_{2}=\int \sum_{j=1}^{N}\left[\left(\zeta|x|^{-1}-\zeta^{\prime}\right)\left|\partial \psi_{j} / \partial r\right|^{2}+\frac{1}{2} \zeta^{\prime}\left|\nabla \psi_{j}\right|^{2}\right] d x
$$

Finally, since the matrix $\left(v_{j k}\right)$ is hermitean, $I_{3}$ can be written as

$$
\begin{aligned}
I_{3}= & -\int \sum_{k, j=1}^{N} \sum_{l=1}^{3} \zeta|x|^{-1} x_{l} v_{k}\left(\partial\left|\psi_{j}\right|^{2} / \partial x_{l}\right) d x \\
& +\operatorname{Re} \int \sum_{k, j=1}^{N} \sum_{l=1}^{3} \zeta|x|^{-1} x_{l} v_{j k} \partial\left(\psi_{j}-\chi_{k}\right) / \partial x_{l} d x .
\end{aligned}
$$

We integrate by parts once more and find that

$$
\begin{aligned}
& I_{3}=\int \sum_{k, j=1}^{N}\left|\psi_{j}\right|^{2}\left[\left(2 \zeta|x|^{-1}+\zeta^{\prime}\right) v_{k}+\zeta \partial v_{k} / \partial r\right] d x \\
& -\operatorname{Re} \int \sum_{k, j=1}^{N} \psi_{j} \overline{\psi_{k}}\left[\left(\zeta^{\prime}+2 \zeta|x|^{-1}\right) v_{j k}+\zeta \partial v_{j k} / \partial r\right] d x .
\end{aligned}
$$

When we combine these expressions we obtain the lemma. 
Following [9] we now choose $\zeta$ as

$$
\zeta(r)=\frac{1}{2}(r+1)^{-1}-1=-(2 r+1) / 2(r+1) .
$$

Then $\zeta \leqq 0, \zeta^{\prime}=-\frac{1}{2}(r+1)^{-2} \leqq 0$ and a direct calculation shows that

$$
\frac{1}{2} \Delta \zeta|x|^{-1}-\zeta^{\prime}|x|^{-2}+\frac{1}{4} \Delta \zeta^{\prime}=(r+4) / 4 r(r+1)^{4}>0 .
$$

We then have

Theorem 1. Let $\psi_{j}, j=1, \ldots, N$, be spherically symmetric solutions of (2) with finite energy, and let $R>0$ be arbitrary. Then for each $j=1,2, \ldots, N$ we have
i) $\int_{0}^{\infty}\left|\psi_{j}(0, t)\right|^{2} d t<\infty$,
ii) $\int_{0}^{\infty} \int_{|x| \leqq R}\left[\left|\psi_{j}\right|^{2}+\left|\partial \psi_{j} / \partial r\right|^{2}\right] d x d t<\infty$,
iii) $\lim _{t \rightarrow \infty} \int_{|x| \leqq R}\left|\psi_{j}\right|^{2} d x=0$.

Proof. Let $T>0$ be arbitrary and integrate (3) over the interval $[0, T]$. We find that

$$
\begin{aligned}
& \left.\operatorname{Im} \sum_{j=1}^{N} \int(2 r+1) 2^{-1}(r+1)^{-1} \psi_{j} \frac{\partial \psi_{j}^{-}}{\partial r} d x\right]_{t=0}^{t=T} \\
& =\frac{1}{2} \sum_{j=1}^{N} \int_{0}^{T} \int(r+1)^{-2}\left|\partial \psi_{j} / \partial r\right|^{2} d x d t \\
& \quad+\pi \sum_{j=1}^{N} \int_{0}^{T}\left|\psi_{j}(0, t)\right|^{2} d t \\
& \quad+\frac{1}{4} \int_{0}^{T} \int \sum_{j=1}^{N}\left|\psi_{j}\right|^{2}(r+4) r^{-1}(r+1)^{-4} d x d t \\
& \quad+\int_{0}^{T} \int \zeta \sum_{k, j=1}^{N}\left[\left|\psi_{j}\right|^{2} \partial v_{k} / \partial r-\operatorname{Re} \psi_{j}{ }_{j} \psi_{k} \partial v_{j k} / \partial r\right] d x d t
\end{aligned}
$$

Let us denote by $L$ the last term appearing here. If we could show that $L \geqq 0$, then, since $\psi_{j}$ and $\partial \psi_{j} / \partial r$ are bounded in $L_{2}\left(\mathbb{R}^{3}\right)$, conclusions i) and ii) of the theorem would follow immediately. To accomplish this, we recall that in the spherically symmetric case the potentials $v_{k}, v_{j k}$ are expressible as

$$
\begin{aligned}
& v_{k}=4 \pi\left[r^{-1} \int_{0}^{r} s^{2}\left|\psi_{k}(s, t)\right|^{2} d s+\int_{r}^{\infty} s\left|\psi_{k}(s, t)\right|^{2} d s\right], \\
& v_{j k}=4 \pi\left[r^{-1} \int_{0}^{r} s^{2} \psi_{j} \psi_{k}(s, t) d s+\int_{r}^{\infty} s \psi_{j} \psi_{k}(s, t) d s\right] .
\end{aligned}
$$

Hence we have

$$
\partial v_{k} / \partial r=-4 \pi r^{-2} \int_{0}^{r} s^{2}\left|\psi_{k}(s, t)\right|^{2} d s
$$


and

$$
\left.\partial v_{j k} / \partial r=-4 \pi r^{-2} \int_{0}^{r} s^{2} \psi_{j} \psi_{k} 7 s, t\right) d s
$$

Thus the expression for $L$ can be written as

$$
(4 \pi)^{-1} L=\int_{0}^{T} \int\left(-\zeta r^{-2}\right)\left[\sum_{j=1}^{N}\left|\psi_{j}\right|^{2} \sum_{k=1}^{N} \int_{0}^{r} s^{2}\left|\psi_{k}\right|^{2} d s-\sum_{j, k=1}^{N} \operatorname{Re} \psi_{j}-\psi_{k} \int_{0}^{r} s^{2} \psi_{j} \psi_{k}-d s\right] d x d t .
$$

Recall that $\zeta \leqq 0$. Let $J$ be the expression in the brackets above. We show that $J \geqq 0$. We have by the Schwarz inequality

$$
\begin{aligned}
J \geqq & \sum_{j=1}^{N}\left|\psi_{j}\right|^{2} \sum_{k=1}^{N} \int_{0}^{r} s^{2}\left|\psi_{k}\right|^{2} d s \\
& -\sum_{j, k=1}^{N}\left|\psi_{j}^{-} \psi_{k}\right|\left(\int_{0}^{r} s^{2}\left|\psi_{j}\right|^{2} d s\right)^{\frac{1}{2}}\left(\int_{0}^{r} s^{2}\left|\psi_{k}\right|^{2} d s\right)^{\frac{1}{2}} \\
\geqq & \sum_{j=1}^{N}\left|\psi_{j}\right|^{2} \sum_{k=1}^{N} \int_{0}^{r} s^{2}\left|\psi_{k}\right|^{2} d s-\left(\sum_{j, k=1}^{N}\left|\psi_{j}\right|^{2} \int_{0}^{r} s^{2}\left|\psi_{k}\right|^{2} d s\right)^{\frac{1}{2}} . \\
& \left(\sum_{j, k=1}^{N}\left|\psi_{k}\right|^{2} \int_{0}^{r} s^{2}\left|\psi_{j}\right|^{2} d s\right)^{\frac{1}{2}} \\
\geqq & 0 .
\end{aligned}
$$

Hence $L$ is nonnegative, which proves i) and ii).

It remains to establish iii). We put

$$
Q_{R}\left[\psi_{j}(t)\right]=\int_{|x| \leqq R}\left|\psi_{j}(x, t)\right|^{2} d x .
$$

From [2] we have the conservation law

$$
\frac{1}{2} \partial\left|\psi_{j}\right|^{2} / \partial t=\operatorname{Im} \sum_{k=1}^{3}\left(\partial / \partial x_{k}\right)\left\{\frac{1}{2} \psi_{j} \partial \psi_{j} / \partial x_{k}-(4 \pi)^{-1} \sum_{l=1}^{N} v_{j l} \partial v_{j l}-/ \partial x_{k}\right\} .
$$

We sum this over $j$ and integrate over $|x| \leqq \varrho$ :

$$
\begin{aligned}
& \frac{1}{2} \sum_{j=1}^{N}(\partial / \partial t) Q_{\varrho}\left[\psi_{j}(t)\right]=\frac{1}{2} \operatorname{Im} \sum_{j=1}^{N} \int_{|x|=\varrho} \psi_{j}-\partial \psi_{j} / \partial r d S_{x} \\
& -\frac{1}{4 \pi} \operatorname{Im} \sum_{j, l=1}^{N} \int_{|x|=\varrho} v_{j l} \partial v_{j l}-\partial r d S_{x} .
\end{aligned}
$$

The last expression vanishes identically since $v_{j l}{ }^{-}=v_{l j}$. This relation is integrated with respect to $\varrho$ over $R_{1}<\varrho<R_{2}$, where $R_{1}>0$; we get

$$
\sum_{j=1}^{N}(\partial / \partial t) \int_{R_{1}}^{R_{2}} Q_{\varrho}\left[\psi_{j}(t)\right] d \varrho=\operatorname{Im} \sum_{j=1}^{N} \int_{R_{1} \leqq|x| \leqq R_{2}} \psi_{j}\left(\partial \psi_{j} / \partial r\right) d x
$$


Next, we integrate this with respect to $t$ over $t_{1} \leqq t \leqq \tau$, obtaining

$$
\begin{aligned}
& \sum_{j=1}^{N}\left(\int_{R_{1}}^{R_{2}} Q_{\varrho}\left[\psi_{j}(\tau)\right] d \varrho-\int_{R_{1}}^{R_{2}} Q_{\varrho}\left[\psi_{j}\left(t_{1}\right)\right] d \varrho\right) \\
& =\sum_{j=1}^{N} \operatorname{Im} \int_{t_{1}}^{\tau} \int_{R_{1} \leqq|x| \leqq R_{2}} \psi_{j}^{-}\left(\partial \psi_{j}(x, s) / \partial r\right) d x d s .
\end{aligned}
$$

This is integrated once again with respect to $\tau$ over $t_{1} \leqq \tau \leqq t$. The result can be written as

$$
\begin{aligned}
& \left(t-t_{1}\right) \int_{R_{1}}^{R_{2}} \sum_{j=1}^{N} Q_{\varrho}\left[\psi_{j}\left(t_{1}\right)\right] d \varrho=\sum_{j=1}^{N} \int_{t_{1}}^{t} \int_{R_{1}}^{R_{2}} Q_{\varrho}\left[\psi_{j}(\tau)\right] d \varrho d \tau \\
& -\operatorname{Im} \int_{t_{1}}^{t}(t-s) \sum_{j=1}^{N} \int_{R_{1} \leqq|x| \leqq R_{2}} \psi_{j}^{-}\left(\partial \psi_{j}(x, s) / \partial r\right)(x, s) d x d s
\end{aligned}
$$

which leads to the inequality

$$
\begin{aligned}
& \left(t-t_{1}\right)\left(R_{2}-R_{1}\right) \sum_{j=1}^{N} Q_{R_{1}}\left[\psi_{j}\left(t_{1}\right)\right] \\
& \leqq\left(R_{2}-R_{1}\right) \int_{t_{1}}^{t} \sum_{j=1}^{N} Q_{R_{2}}\left[\psi_{j}(\tau)\right] d \tau \\
& \quad+\frac{1}{2} \sum_{j=1}^{N} \int_{t_{1}}^{t}(t-s) \int_{R_{1} \leqq|x| \leqq R_{2}}\left(\left|\psi_{j}\right|^{2}+\left|\partial \psi_{j} / \partial r\right|^{2}\right) d x d s .
\end{aligned}
$$

We now choose $t_{1}=t-1$ and apply ii) to conclude that iii) is valid.

We remark that the assumption of spherical symmetry was only needed to show that the last term in the expression (3) was nonnegative (for $\zeta<0$ ).

For the single Equation (1) no such assumption is necessary. In fact, in this case we have the following identity:

Lemma. A solution $\psi$ of finite energy of (1) satisfies the identity

$$
\begin{aligned}
& -(d / d t) \operatorname{Im} \int \zeta \psi \psi_{r}-d x=-\int \zeta^{\prime}\left|\psi_{r}\right|^{2} d x \\
& -\int \zeta|x|^{-1}\left(|\nabla \psi|^{2}-\left|\psi_{r}\right|^{2}\right) d x-2 \pi \zeta(0)|\psi(0, t)|^{2} \\
& +\int|\psi|^{2}\left[\frac{1}{2} \Delta \zeta|x|^{-1}-\zeta^{\prime}|x|^{-2}+\frac{1}{4} \Delta \zeta^{\prime}\right] d x \\
& -(8 \pi)^{-1} \int \zeta^{\prime}|\nabla v|^{2} d x-(4 \pi)^{-1} \int\left(\zeta|x|^{-1}-\zeta^{\prime}\right)(\partial v / \partial r)^{2} d x,
\end{aligned}
$$

for $\zeta$ as above.

Now again choose $\zeta<0$ as in (4). Since $\zeta^{\prime}<0$ and since by direct calculation $\zeta|x|^{-1}-\zeta^{\prime}=-\left(2 r^{2}+2 r+1\right) / 2 r(r+1)^{2} \leqq 0$

we conclude, as in ii) of Theorem 1 , that for any $R>0$,

$$
\int_{0}^{\infty} \int_{|x| \leqq R}\left[|\psi|^{2}+|\partial \psi / \partial r|^{2}\right] d x d t<\infty .
$$


To bound the gradient of $\psi$, we put $\zeta \equiv-1$ in (5) and obtain

$$
\begin{aligned}
& -(d / d t) \operatorname{Im} \int \psi \psi_{r}{ }^{-} d x=\int|x|^{-1}\left(|\nabla \psi|^{2}-\left|\psi_{r}\right|^{2}\right) d x+2 \pi|\psi(0, t)|^{2} \\
& +(4 \pi)^{-1} \int|x|^{-1} v_{r}{ }^{2} d x .
\end{aligned}
$$

Thus by (6), $\int_{0}^{\infty} \int_{|x| \leqq R}|\nabla \psi|^{2} d x d t$ is finite. Hence we have

Theorem 2. Let $\psi$ be a solution of (1) with finite energy. Then
i) $\int_{0}^{\infty}|\psi(0, t)|^{2} d t<\infty$
ii) $\int_{0}^{\infty} \int_{|x| \leqq R}\left[|\psi|^{2}+|\nabla \psi|^{2}\right] d x d t<\infty$.
iii) $\int_{0}^{\infty} \int_{\mathbb{R}^{3}}|x|^{-1} v_{r}^{2} d x d t<\infty$
iv) $\lim _{t \rightarrow \infty} Q_{R}[\psi(t)]=\lim _{t \rightarrow \infty} \int_{|x| \leqq R}|\psi|^{2} d x=0$.

Of course, iii) is the direct analogue of Morawetz' estimate [5] (cf. also [6]). Conclusion iii) seems to be very weak here. In fact, it is not difficult to show, by considering the spherical means of $v$ that iii) implies

$$
\int_{0}^{\infty} Q_{R}^{2}[\psi(t)] d t<\infty
$$

which is a trivial result in view of ii), iv) above.

\section{Nonexistence of Scattering}

Consider again the equation

$i \partial \psi / \partial t=\Delta \psi-v(\psi) \psi$

where

$$
v(\psi)=\int|x-y|^{-1}|\psi(y, t)|^{2} d y
$$

and where we have changed the coefficient of $\Delta \psi$ to unity for simplicity. We call a solution $\psi$ of $\left(1^{\prime}\right)$ asymptotically free if there exists a free solution $\psi_{+}$(a solution of the linear Schrödinger equation) satisfying

$$
\left\|\psi(t)-\psi_{+}(t)\right\|_{2} \rightarrow 0 \quad \text { as } \quad t \rightarrow \infty .
$$

We assume the solution $\psi$ itself has data in $L_{2}$ and that the free state $\psi_{+}$has data in $L_{1} \cap L_{2}$.

Following [3], [7] we then have

Theorem 3. The only solution of $\left(1^{\prime}\right)$ which is asymptotically free is $\psi \equiv 0$. 
Proof. Suppose that $\psi \neq 0$ is a solution of $\left(1^{\prime}\right)$ which has an asymptotic free state $\psi_{+}$. We form the expression

$$
H(t)=\operatorname{Im} \int \psi \psi_{+}-d x
$$

and calculate directly

$$
\begin{aligned}
\dot{H}(t)= & \operatorname{Re} \int v(\psi) \psi \psi_{+}-d x \\
= & \int v\left(\psi_{+}\right)\left|\psi_{+}\right|^{2} d x+\int\left|\psi_{+}\right|^{2}\left(v(\psi)-v\left(\psi_{+}\right)\right) d x \\
& +\operatorname{Re} \int \psi_{+}-v(\psi)\left(\psi-\psi_{+}\right) d x .
\end{aligned}
$$

Put

$$
\begin{aligned}
& J_{1}=\int\left|\psi_{+}\right|^{2}\left(v(\psi)-v\left(\psi_{+}\right)\right) d x \\
& J_{2}=\operatorname{Re} \int \psi_{+}-v(\psi)\left(\psi-\psi_{+}\right) d x .
\end{aligned}
$$

As is well-known, $\psi_{+}$satisfies the estimate

$$
\left\|\psi_{+}(t)\right\|_{\infty}=0\left(t^{-3 / 2}\right) \text { as } t \rightarrow \infty .
$$

Hence we have, for $t \geqq 1$, say,

$$
\begin{aligned}
\left|J_{1}\right| & \leqq\left\|\psi_{+}(t)\right\|_{\infty}^{2 / 3} \int\left|\psi_{+}\right|^{4 / 3}\left|v(\psi)-v\left(\psi_{+}\right)\right| d x \\
& \leqq \text { const. } t^{-1}\left\|\psi_{+}^{4 / 3}(t)\right\|_{3 / 2}\left\|v(\psi)-v\left(\psi_{+}\right)\right\|_{3} \\
& \leqq \text { const. } t^{-1}\left\|\psi_{+}(t)\right\|_{2}^{4 / 3}\left\|\psi(t)-\psi_{+}(t)\right\|_{2}
\end{aligned}
$$

where we have used a Sobolev-type inequality from [1] to estimate the $L_{3}$-norm of the expression $v(\psi)-v\left(\psi_{+}\right)$. Thus

$$
\left|J_{1}\right|=o\left(t^{-1}\right) \text { as } t \rightarrow \infty \text {. }
$$

Similarly we have

$$
\begin{aligned}
\left|J_{2}\right| & \leqq\left\|\psi_{+}(t)\right\|_{\infty}^{2 / 3} \int\left|\psi_{+}\right|^{1 / 3} v(\psi)\left|\psi-\psi_{+}\right| d x \\
& \leqq \text { const. } t^{-1}\left\|\psi_{+}^{1 / 3}(t)\right\|_{6}\|v(\psi)(t)\|_{3}\left\|\psi(t)-\psi_{+}(t)\right\|_{2} \\
& \leqq \text { const. } t^{-1}\left\|\psi_{+}(t)\right\|_{2}^{1 / 3}\|\psi(t)\|_{2}\left\|\psi(t)-\psi_{+}(t)\right\|_{2} \\
& =o\left(t^{-1}\right) \quad \text { as } \quad t \rightarrow \infty
\end{aligned}
$$

Hence from (7) we have

$$
\dot{H}(t) \geqq \int v\left(\psi_{+}\right)\left|\psi_{+}\right|^{2} d x-o\left(t^{-1}\right)
$$

for all $t \geqq 1$, say. Now let $k>0$ be arbitrary. Then

$$
v\left(\psi_{+}\right)=\int|x-y|^{-1}\left|\psi_{+}(y, t)\right|^{2} d y \geqq \int_{|y|<k t}|x-y|^{-1}\left|\psi_{+}(y, t)\right|^{2} d y .
$$

Hence for $|x|<k t$ we certainly have

$$
v\left(\psi_{+}\right) \geqq(2 k t)^{-1} \int_{|y|<k t}\left|\psi_{+}(y, t)\right|^{2} d y .
$$


It follows that

$$
\begin{aligned}
\int v\left(\psi_{+}\right)\left|\psi_{+}\right|^{2} d x & \geqq \int_{|x|<k t} v\left(\psi_{+}\right)\left|\psi_{+}\right|^{2} d x \\
& \geqq(2 k t)^{-1}\left(\int_{|x|<k t}\left|\psi_{+}(x, t)\right|^{2} d x\right)^{2} .
\end{aligned}
$$

However, from [7] we have the result

$$
\lim _{t \rightarrow \infty} \int_{|x|<k t}\left|\psi_{+}\right|^{2} d x=\int_{|\xi|<k / 2}\left|\varphi_{+} \hat{(}(\xi)\right|^{2} d \xi
$$

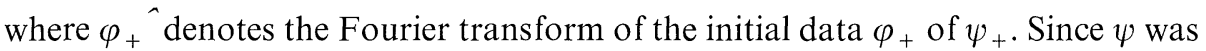
assumed nontrivial, there is some value of $k$ for which this limit does not vanish. Then from (8) we have that there exists a positive constant $c_{0}$ such that

$$
\dot{H}(t) \geqq c_{0} / t
$$

for all sufficiently large $t$. Since the left-hand side here is integrable in time, we have a contradiction, which proves the theorem.

We remark finally that for solutions of Equation (2), a simple corresponding theorem does not seem to be readily available. This is due to the fact that the dominant term in the proof of Theorem 3 vanishes identically in equations (2), i.e. when $k=j$ in Equation (2), the resulting nonlinear term disappears.

\section{References}

1. Bers, John, Schecter: Partial differential equations. New York: Interscience 1964

2. Chadam,J.M., Glassey,R.T.: Global existence of solutions to the Cauchy problem for timedependent Hartree equations. J. Math. Phys. 16, 1122-1130 (1975)

3. Glassey, R. T.: On the asymptotic behavior of nonlinear wave equations. Trans. Amer. Math. Soc. 182, 187-200 (1973)

4. Lin,J.-E.: Time decay for two conservative equations. Ph.D. Thesis, Brown University, 1976

5. Morawetz, C.S. : Time decay for the nonlinear Klein-Gordon equation. Proc. Roy. Soc. A 306, 291$296(1968)$

6. Morawetz,C.S., Strauss,W.A.: Decay and scattering of solutions of a nonlinear relativistic wave equation. Comm. Pure Appl. Math. 25, 1-31 (1972)

7. Strauss,W. A. : Nonlinear scattering theory; in Proc. of NATO Advanced Study Inst., June 1973: Scattering theory in mathematical physics, $53-78$

8. Strauss, W. A.: Dispersal of waves vanishing on the boundary of an exterior domain. Comm. Pure. Appl. Math. 28, 265-278 (1975)

9. Strauss, W. A., Cooper,J.: Energy boundedness and decay of waves reflecting off a moving obstacle. University of Maryland Technical Report TR-74-69, 1974

Communicated by J. Glimm

Received October 25, 1976 